PREPARED FOR SUBMisSion to JHEP

\title{
Dynamical Quarkonia Suppression in a QGP-Brick
}

\author{
Jorge Casalderrey-Solana \\ Departament d'Estructura i Constituents de la Matèria and Institut de Ciències del Cosmos (IC- \\ CUB), Universitat de Barcelona, Martí i Franquès 1, 08028 Barcelona, Spain \\ E-mail: jorge.casalderrey@ub.edu
}

\begin{abstract}
I address the effect that a temperature dependent potential has on the suppression of heavy quarkonia states in deconfined hadronic matter. I focus on a simple medium: a homogenous, fixed temperature and deconfined system with a finite lifetime (QGP-brick). Assuming that all the interactions of a heavy quark anti-quark $(Q-\bar{Q})$ pair with the medium can be recast into an in-medium potential, I solve the time dependent Schrödinger equation to evolve the density matrix which describes the hard pair production and its connection to the final distribution of hadrons after the medium disappears. For those temperatures in which bound states survive in the medium, I find a non-trivial dependence of the production of excited quarkonia states on the in-medium levels, due to the mixing of vacuum and in-medium wave functions. This mixing leads, in particular, to the enhancement of the relative abundance of $2 \mathrm{~S}$ to $1 \mathrm{~S}$ states for those systems in which the in-medium ground state of the $Q-\bar{Q}$ system is dissolved or close to threshold. I also explore quarkonia production in a non-homogeneous expanding medium and find that the finite formation time effects arising from the low binding energies of in-medium states lead to the insensitivity of the heavy mesons yield to the hottest part of the system evolution.
\end{abstract}




\section{Contents}

1 Introduction 1

2 Two Simple Models for the in-Medium Potential 3

3 The QGP-Brick $\quad 6$

3.1 Quarkonia Production from a Hard Process 6

$\begin{array}{ll}3.2 & \text { Quarkonia Suppression in a QGP-Brick }\end{array}$

4 Quarkonia Suppression in an Expanding Medium 12

5 Quarkonia Suppression in a non-Homogenous Expanding Medium $\quad 14$

$\begin{array}{llr}6 & \text { Discussion } & 17\end{array}$

\section{Introduction}

At temperatures of about $T_{c} \approx 175 \mathrm{MeV}$, QCD experiences a rapid cross over transition into a deconfined state of matter, the Quark Gluon Plasma (QGP). While at asymptotically high temperatures the QGP behaves as a free gas of quarks and gluons, in the vicinity of $T_{c}$ strong interactions among the colored plasma constituents remain, which complicate the description of the medium dynamics. These interactions make the QGP an extremely rich system, with strong collective phenomena which arise directly from an non-abelian fundamental gauge theory. Therefore, the characterization of this region of the QCD phase diagram has been the subject of intense theoretical and experimental research.

From the experimental point of view, this state of matter is studied by the ultrarelativistic collision of heavy ions which form a hot and dense hadronic medium. At the collision energies explored at RHIC and the LHC, the energy density of this medium is sufficiently large to reach the deconfined phase at the early stage of the system evolution [13]. One of the main tools available for determining the medium dynamics is its interactions with hard probes. Among those, an important set is the different heavy quarkonia states and their in-medium modifications [4]. The advantage of these probes is that the heavier their constituents quarks are, the smaller the distances they probe so that their properties can be described within a peturbative QCD framework and, thus, are under good theoretical control. The interaction of these states with the medium leads to a modification of those properties and even to their complete dissociation at sufficiently high temperatures, which, in turn, leads to a suppression of quarkonium yields in nuclear collisions as compared to their p-p counterpart.

Traditionally, due to the small b-quark production cross section at low collision energies, most studies have focussed on charmonia states (such as the $\mathrm{J} / \psi$ ). However, charm quarks 
are not sufficiently heavy for a reliable theoretical description, which leads to significant uncertainties both in their production mechanism in nuclear collisions [5] as well as their interactions with deconfined matter (see, [6] for a recent review), what complicates data interpretations. In addition, the abundance of $c-\bar{c}$ produced in the collision also leads to recombination effects [7-10] which also obscure the use of these states as medium probes. Therefore, while $J / \psi$ states suffer a strong suppression in hadronic collisions [11-16], the suppression pattern is still puzzling; a particularly surprising example of these puzzles is the recent preliminary data of $\psi(2 S)$ production in $\mathrm{Pb}-\mathrm{Pb}$ collisions at the LHC [17] which show an abnormal enhancement of $\psi(2 S)$ relative to $J / \psi$ states, which seem at odds with the sequential dissociation of the different quarkonia levels. Fortunately, data on bottomonium suppression have recently become available both at the LHC [16] and at RHIC [18] opening a much more theoretically controlled channel.

The theoretical description of in-medium quarkonia properties has also experienced significant progress in recent years. Contrary to early expectations, current lattice analyses of charm and bottom current-current correlators indicate that quarkonia states survive the deconfinement phase transition up to a temperature slightly higher than $T_{c}$ [19-25]. Although the determination of chamonium and bottomonium spectral functions is still under intense theoretical investigation, there is a consensus that, at least, the bottomonium ground states survive to a temperature as high as $T=2 T_{c}$, which is comparable to the highest temperatures reached at the LHC, while other excited states dissolve at a lower temperature. The situation for the in-medium $J / \psi$ is less clear, but indications from the lattice suggest that it is totally dissolved at $T<1.5 T_{c}[25]$.

Complementary to lattice studies, potential models have been extensively used to describe the properties of finite-temperature quarkonia states [26-36]. In this approach the interactions among the heavy quarks in the bound states are encoded by an inter-quark potential and the different estates correspond to the non-relativistic levels of the corresponding Schrödinger equation. In the vacuum, since the potential can be directly extracted from lattice calculations [37], this approach provides a good description of the different vacuum quarkonia states [38]. In the medium, the main assumption is that all the interaction of the heavy pair with the plasma degrees of freedom can be codified in a medium-modified potential. However, contrary to the vacuum case, this temperature dependent potential is not easy to extract from the lattice $[39,40]$ and most studies rely on phenomenological potentials adjusted to describe lattice correlators. In recent years, effective field theory approaches have put this potential description in firm theoretical grounds [41-43], clarifying the region of validity of the potential assumption at finite temperature. The explicit determination of the potential at sufficiently high temperature, such that the plasma dynamics are perturbative, have, in fact, led to the unexpected conclusion that in-medium potentials develop imaginary parts as a result of the destruction by the plasma of singlet $Q-\bar{Q}$ contributions $[39,40]$.

The in-medium properties of quarkonia extracted from the static studies above serve as an input for dynamical models of quarkonia production in ultra relativistic collisions. Over the last years the description of $J / \psi$ production in these collisions has led to a great body of work which accounts for a large variety of physical processes of relevance to this 
production (see [6] and references therein for a comprehensive review). More recently, the LHC capabilities for a detailed study of the $\Upsilon$ family suppression have led to several studies focussed on these mesons [44-47]. In this work I will also address the suppression of heavy mesons by focussing on the effect a temperature dependent potential has on the production of quarkonia states in a deconfined hadronic matter. For simplicity, I will restrict myself to a deconfined medium with a fixed temperature which interacts with a heavy $Q-\bar{Q}$ pair during a finite time (QGP-brick). The pair is generated by a generic hard process and I will assume that the production is dominated by color singlet configurations. Contrary to other approaches, I will not rely on adiabatic approximations for the in-medium states, but I will address the suppression dynamically by solving the time dependent Schrödinger equation without assuming the dominance of any in-medium state.

The paper is organized as follows: In section 2 I describe the two simplified sample potentials which I used in substitution of the unknown in-medium potential in the region of interest and which mimic some of the main features expected from this potential. In section 3 I describe the QGP-brick set-up. The formalism I have used to connect the hard production to the solutions of the time-dependent Schrödinger equation is described in subsection 3.1 and in subsection 3.2 I solve numerically the time dependent Schrödiger equation and compare the quarkonia production in vacuum and in medium for $1 \mathrm{~S}$ and $2 \mathrm{~S}$ states. Subsequently, in sections 4 and 5, having in mind heavy ion physics application, I study the effect of a time and spatial variation of the in-medium potential. Finally, I discuss the main findings of this study in section 6 .

\section{Two Simple Models for the in-Medium Potential}

Potential models have long been used to describe the properties of different quarkonia states in QCD plasma [26-35]. As already mentioned, the main assumption of this approach is that the interactions of the $Q-\bar{Q}$ pair with the thermal medium can be recast into a temperature dependent in-medium potential. While in recent years effective theory methods have led to a theoretical justification of this phenomenological approach in a certain limit $\left(1 / r_{B} \sim \mu_{D} \gg E_{b}\right.$, with $r_{B}$ and $E_{b}$ the Bohr radius and binding energy of the state and $\mu_{D}$ the Debye screening length of the plasma) [41-43], the functional form of the thermal potential for temperatures slightly higher than $T_{c}$ remains unknown. Several studies based on phenomenologically motivated potentials have led to a remarkably good agreement with lattice data on heavy quark correlation functions [33-35]. In most of these analyses, the in-medium potential is taken to be real and somewhat related to the singlet free energy computed via lattice QCD. However, in recent years perturbation theory analyses have shown that the in-medium potential does not need to be real since the interaction with the colored medium can change the color state of the pair, effectively reducing the probability of the pair to remain in a color singlet state [39, 40]. While for the region of interest the perturbative expression for the potential is not reliable since in the vicinity of $T_{c}$ the coupling is not small, the many body effect described above exist beyond a perturbative treatment and leads to an imaginary part for the singlet potential, which leads the disappearance of singlet states in plasma. 

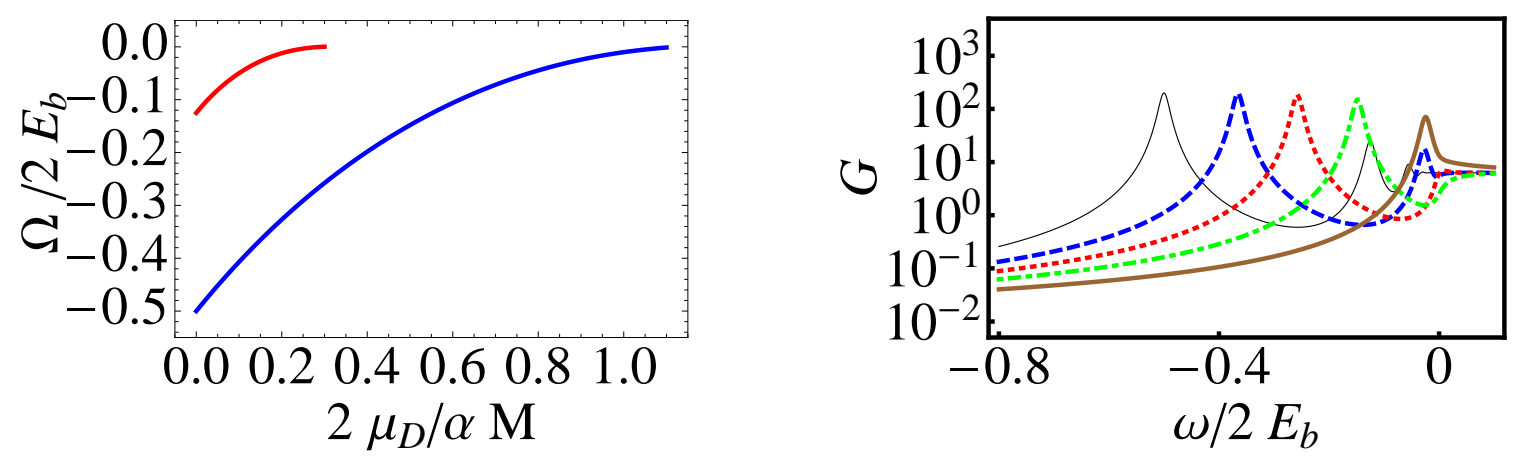

Figure 1. Left: Binding energy of the 1S (lower curve) and 2S (upper curve) states of the Yukawa potential, eq. (2.1), in units of the vacuum ground state anergy, $E_{b}=\alpha^{2} M / 4$ as a function of the Debye screening length $\mu_{D}$. Right: Spectral function associated to the Yukawa potential (in arbitrary units) as a function of the energy $\omega$, for different values of $2 \mu_{D} / \alpha M=0,0.15,0.3,0.5,0.9$ for the thin, dashed, dotted, dash dotted and solid lines respectively.

Given the absence of an explicit form of the in-medium potential for the region of interest, in this work I will focus on the effect on quarkonia production of two toy-model potentials which mimic some of the most important features of the in-medium dynamics without any attempt to reconcile these potentials to lattice computations of the spectral function. Since this approach will already prevent me from a direct comparison with quarkonia suppression data on heavy ion collisions, I will further assume, for simplicity, that the vacuum quarkonia states are described by coulombic wave functions; while this is a reasonable approximation for sufficiently heavy quarks, significant deviations are found for realistic quark masses.

Since the relative magnitude of the imaginary part of the potential in the vicinity of $T_{c}$ is currently unknown, I will explore a (real) Yukawa potential, which encodes the color screening effects in the plasma.

$$
V_{R}(r)=-\alpha \frac{e^{-\mu_{D} r}}{r},
$$

with $\mu_{D}$ the Debye screening length of the plasma, which characterizes the thermal state and $\alpha$ an adjustable parameter. This potential coincides with the real part of the perturbative potential, up to a constant which is irrelevant for the temporal evolution. According to our assumption on the vacuum wave functions, we will consider the coulomb potential $\mu_{D}=0$ as the vacuum potential.

The screening of the quark color charge in the plasma leads to the disappearance of the in-medium quarkonia states. By solving the eigenvalue problem for different values of $\mu_{D}$, the biding energy of the ground state and the first excited state are determined. Their binding energies as a function of $\mu_{D}$ are shown left panel of fig. (1). According to general expectations, excited states dissolved at lower plasma temperatures than the ground state. In the right panel of fig. (1) I show the spectral function $G$ of the corresponding Schrödinger equation (see [33] for details on how to compute this spectral function). For illustration porpoises, we have evaluated it at $\omega=E-i \epsilon$ with $\epsilon=0.02 E_{b}$ and $E_{b}=\alpha^{2} M / 4$ 

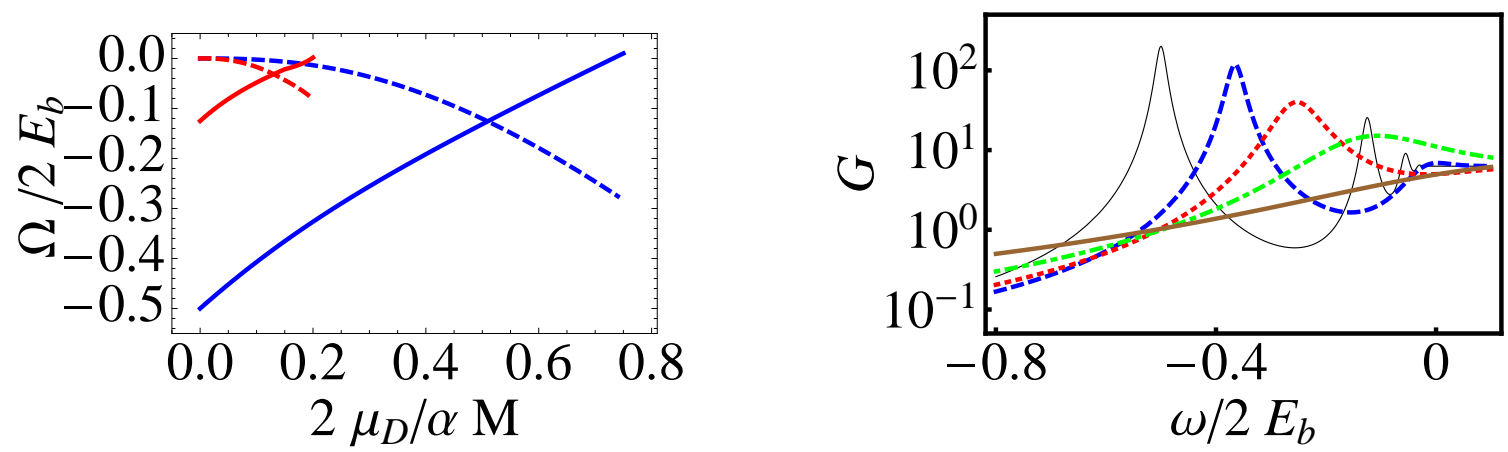

Figure 2. Left: Real (solid) and imaginary (dashed) part of the binding energy of the 1S (lower curve) and $2 \mathrm{~S}$ (upper curve) states of the complex potential, eq. (2.2) $\mathrm{m}$ in units of the vacuum ground state anergy, $E_{b}=\alpha^{2} M / 4$ as a function of the Debye screening length $\mu_{D}$. Right: Spectral function associated to the same potential (in arbitrary units) as a function of the energy $\omega$, for different values of $2 \mu_{D} / \alpha M=0,0.15,0.3,0.5,0.9$ for the thin, dashed, dotted, dash dotted and solid lines respectively.

the binding energy of the vacuum ground state. At zero temperature (thin solid line), the spectral function shows several energy levels (the higher $\mathrm{n}$ excitations overlap because of the finite value of $\epsilon$ ). As the Debye screening length increases, the excited states disappear and the ground state moves towards threshold. The reduction of the biding energy is accompanied by a reduction of the spectral strength, which coincides with a reduction of the modulus of the in-medium wave function at the origin. Quarkonia states are, thus, expected to be suppressed in the medium even if they remain bound at finite temperature.

In order to study the effect of a complex in-medium potential, I also analyze a potential which differs from the Yukawa one, eq. (2.1), by an imaginary part

$$
V_{I}(r)=-\alpha\left(\frac{e^{-\mu_{D} r}}{r}+i \mu_{D} \phi\left(\mu_{D} r\right)\right)
$$

with

$$
\phi(r)=\int_{0}^{\infty} d z \frac{z}{\left(z^{2}+1\right)^{2}}\left(1-\frac{\sin (z r)}{z r}\right),
$$

which coincides with the functional form of the imaginary part of the perturbative potential $[39,40]$. In comparing with the potential in $[39,40]$ the reader may notice a different normalization of the imaginary contribution in eq. (2.2). While in perturbation theory the prefactor of $\phi$ is given by the temperate scale, T, in this work, for simplicity of the analysis, I have used $\mu_{D}$ instead. Since in the region of interest we expect $\mu_{D} \lesssim T$, this expression will give a reasonable estimate of the effect of the imaginary part on quarkonia suppression. In the rest of this paper, I will always assume that $\mu_{D}$ is of order $T$ and use one or another simultaneously.

Thermal effects for this potential are twofold. The screening of the quark color charge leads to the disappearance of bound levels as in the previous case; additionally, the complex potential introduces an imaginary part to the poles of the spectral density. Thus, the eigenvalue problem for the potential in this case leads to complex eigenstates; in the right 
hand side of fig. (2) I show the real and imaginary part of the ground and first excited states. The comparison of the real part of the biding energy with those of the real potential, fig. (1), shows that the complex potential is more effective in dissolving the bound states; additionally, the imaginary part of the eigenstates grows and becomes as large as the real one well before the bound state is dissolved. These imaginary eigenvalues are reflected in the broadening of the peak structures in the spectral density shown in fig. (2). The spectral density also shows that, in addition to the faster level disappearance, the spectral strength of the state is reduced faster for the imaginary potential than for the real one; thus, we expect that the complex potential eq. (2.2) will lead to a stronger quenching of quarkonia states than the real potential eq. (2.1).

\section{The QGP-Brick}

The spectral functions computed in the previous section encode the distribution of quarkonia states at fixed temperature (or $\mu_{D}$ ) in equilibrium. If the medium described by the potentials eq. (2.2) and eq. (2.1) would be sufficiently large and exist for a sufficiently long time, the asymptotic distribution of quarkonia states would be a thermal distribution according to that spectral function and independent of the origin of the $Q-\bar{Q}$. Furthermore, for these long media, the dilepton rate in the region of invariant mass of order $2 M$ would be directly related to those spectral functions. However, for any realistic application the medium is finite and short-lived. Thus, the study of not only the pre-asymptotic dynamics but also the modification of the formation of the long-lived vacuum quarkonia states by the presence of a finite medium are of relevance for the understanding of quarkonia production in a heavy ion environment.

To study those processes, I will first study quarkonia production in a homogeneous medium at fixed temperature with a finite lifetime after which the temperature drops abruptly to zero. The spacial extent of the medium is taken to be large, much larger that the in-medium radius of bound states. I will assume that a $Q-\bar{Q}$ pair is formed within the medium via a hard process at a time $t_{0}=0$ and interacts with it during a finite time $t$ after which the medium disappears. After this time, the evolution occurs as

in vacuum. In analogy with the jet quenching studies, where a similar set-up was used to analyze the differences among the available quenching models, I will call this set-up, which I have sketched in fig. (3), a QGP-brick.

\subsection{Quarkonia Production from a Hard Process}

I focus in the production of quarkonia states from a hard process which takes place within the QGP-brick. For sufficiently heavy quarks $\left(M_{q} \gg \Lambda_{Q C D}\right)$, this process can be factorized into a short distance behavior, described via perturbation theory, and a long distance, non perturbative matrix element. The production cross section of a state $\mathrm{S}$ is expressed as [48]

$$
d \sigma(S)=\sum_{n} d \sigma(Q \bar{Q}([n]))\langle Q \bar{Q}([n]) \rightarrow S\rangle,
$$

where $d \sigma(Q \bar{Q}([n]))$ is the perturbative production cross section of a $Q-\bar{Q}$ pair with quantum numbers $n$ and $\langle Q \bar{Q}([n]) \rightarrow S\rangle$ is the soft matrix element which projects a given $Q-\bar{Q}$ 


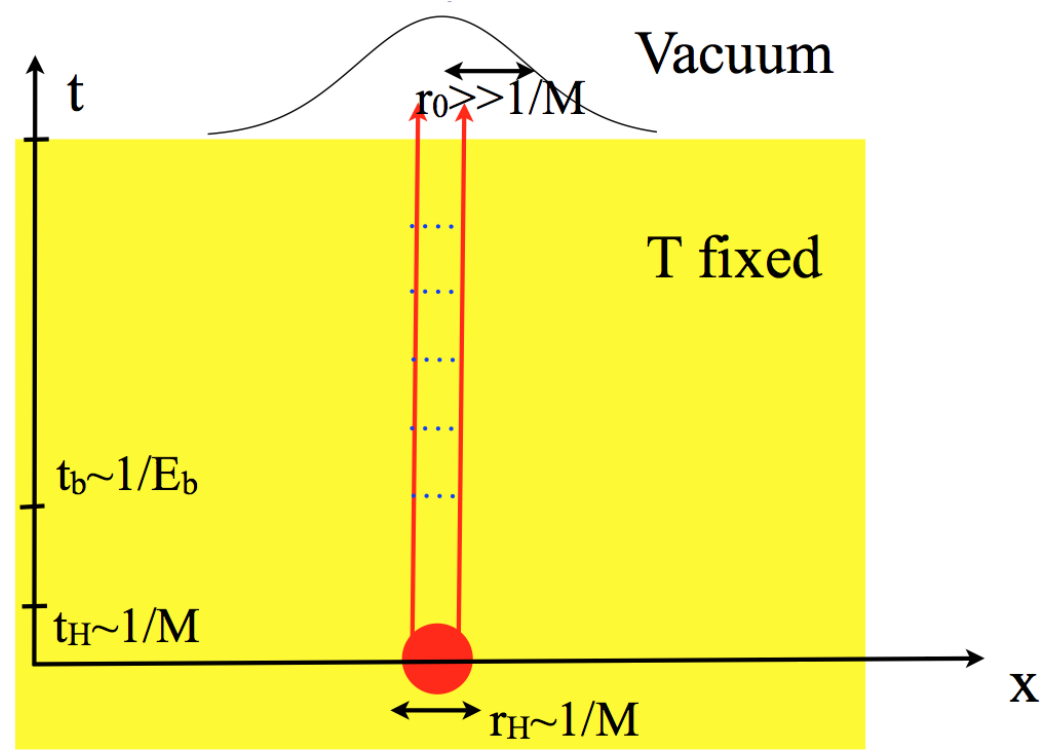

Figure 3. Sketch of the QGP-Brick set up. At initial time a $Q-\bar{Q}$ pair is formed in the medium via a hard process that occurs within a short time $t_{H} \sim 1 / M$. At a later time of order the inverse binding energy, the soft interactions lead to the formation of quarkonia states from the pair, which are influenced by the presence of the medium. At a later time $t$, the medium disappears and the $Q-\bar{Q}$ system projects into vacuum quarkonia states.

pair into the state $\mathrm{S}$. The set of quantum numbers $n$ does not need to coincide with the quantum numbers of the final state. However, for sufficiently heavy quarks, for which the relative velocity v of the $Q-\bar{Q}$ pair is very small, the contribution of those configurations with quantum numbers different from those of the state $\mathrm{S}$ are suppressed by powers of $v$. Therefore, in the rest of the paper I will neglect those corrections ${ }^{1}$. In the particular case of vector S-wave states (such us $J / \psi$ or $\Upsilon$ ) the production cross section is reduced to

$$
d \sigma(S) \approx d \sigma\left(Q \bar{Q}\left(\left[{ }^{3} S_{1}\right]\right)\right)\left\langle Q \bar{Q}\left(\left[{ }^{3} S_{1}\right]\right) \rightarrow S\right\rangle .
$$

I will also assume that the heavy quarks are much heavier than any medium scale, in particular, $M \gg T$. Thus, since the hard part of the production cross section takes place within a space-time region of typical size $1 / M$, which is, by assumption, much smaller than any medium scale, the presence of the plasma cannot alter this part of the production process, and $d \sigma\left(Q \bar{Q}\left(\left[{ }^{3} S_{1}\right]\right)\right)$ remains the same irrespectively of wether the production takes place in the vacuum or within a medium. ${ }^{2}$ Within this approximation, plasma effects are only present in the late time projection of the $Q-\bar{Q}$ pair into the particular quarkonia state. For sufficiently heavy quarks, potential non-relativistic QCD (pNRQCD), which describes the dynamics of soft $M v^{2}$ modes can be used to determine these matrix elements.

\footnotetext{
${ }^{1}$ These include the contribution of color octet states; I am, thus, restricting myself to a color singlet model of quarkonia production.

${ }^{2}$ With this approximation I am also neglecting quark energy loss effects on quarkonia production. While these do not change significantly the production rate, they can alter the momentum distribution of the observed states.
} 
In this approach, the quarkonia dynamics are described via the solution of the Schrödinger equation with a given inter-quark potential and the matrix element is determined from the $Q-\bar{Q}$ pair wave function.

For central $Q-\bar{Q}$ potentials, the center of mass dynamics of the pair decouples and the matrix element is only dependent on the equivalent one body problem. From the point of view of the soft dynamics, the hard production can be described via a Wigner function for the $Q-\bar{Q}$ pair, $W_{H}(\mathbf{r}, \mathbf{q})$, with $\mathbf{r}$ and $\mathbf{q}$ the relative position and momentum of the pair at an initial time $t_{0}$ within the box. Due to the uncertainty principle, $t_{0}$ cannot be determined with an accuracy larger than $1 / M$ but, since I have assumed that this scale is much smaller than any medium or bound state scale, I will neglect this uncertainty. From the Wigner function, the initial one body density matrix is given by

$$
\rho_{H}\left(\mathbf{r}-\frac{\mathbf{y}}{2}, \mathbf{r}+\frac{\mathbf{y}}{2} ; t_{0}\right)=\int \frac{d \mathbf{q}}{(2 \pi)^{3}} e^{i \mathbf{q} \mathbf{y}} W_{H}(\mathbf{r}, \mathbf{q}) .
$$

Independently of the particular process that leads to the production of a $Q-\bar{Q}$ pair within the medium, since the process is hard, the typical momentum of each of the quarks, $p$, is large $M \lesssim p$. Thus, the typical relative quark momentum is also much larger than the inverse bound state radius and, from the point of view of the soft matrix element, only the $q \rightarrow 0$ limit of $W_{H}$ is relevant for the production of bound states, which leads to an approximate initial density matrix given by

$$
\rho_{H}\left(\mathbf{r}-\frac{\mathbf{y}}{2}, \mathbf{r}+\frac{\mathbf{y}}{2} ; t_{0}\right)=\rho_{0}(\mathbf{r}) \delta(\mathbf{y})
$$

Furthermore, since the hard process takes place at very small distances I will approximate the function $\rho_{0}(\mathbf{r})$ by a $\delta$-function at the origin, $\rho_{0}(\mathbf{r}) \propto \delta(\mathbf{r})$.

As the $Q-\bar{Q}$ pair propagates, the density matrix eq (3.4) evolves via a non-relativistic hamiltonian, since the late time interactions are soft as compared to the pair mass. At any given time $t>t_{0}$ the different particle yields can be obtained by projecting the evolved density matrix into the single state density matrix

$$
Y_{S} \propto \operatorname{Tr}\left(\rho_{H}(t) \rho_{s}\right)=\operatorname{Tr}\left(\rho_{H}\left(t_{0}\right) \rho_{s}\left(t-t_{0}\right)\right),
$$

with $\rho_{s}=|S\rangle\langle S|$ and $|S\rangle$ the quarkonium estate of interest. In the second equality above I have used the cyclicity of the trace to define

$$
\rho_{s}\left(t-t_{0}\right)=\left(e^{-i H\left(t-t_{0}\right)}\right)^{\dagger}|S\rangle\left\langle S\left|\left(e^{-i H\left(t-t_{0}\right)}\right)=\right| \tilde{S}\right\rangle\langle\tilde{S}|
$$

where $|\tilde{S}\rangle$ is the (backwards in time) evolved wave function. Taking $t_{0}$ as the evolution variable and performing the change $\tilde{t}=t-t_{0}$ this wave function satisfies the (hermitian conjugate) Schrödinger equation:

$$
-i \partial_{\tilde{t}} \tilde{\psi}(\tilde{t}, \mathrm{r})=-\frac{\nabla^{2}}{M_{q}} \tilde{\psi}(\tilde{t}, \mathrm{r})+V^{\dagger}(r) \tilde{\psi}(\tilde{t}, \mathrm{r})
$$

with $\tilde{\psi}(\tilde{t}, \mathrm{r})=\langle\mathbf{r} \mid \tilde{S}\rangle$ and initial condition $\tilde{\psi}(0, \mathrm{r})=\langle\mathbf{r} \mid S\rangle$. 
Combining eq. (3.4), eq. (3.5) and eq. (3.6), the yield of quarkonia production after a time of propagation $t$ is given by

$$
Y_{S} \propto\left|\tilde{\psi}\left(t-t_{0}, \mathbf{r}=0\right)\right|^{2}
$$

In the vacuum, the inter quark potential is hermitian and the modulus of the the wave function at the origin remains constant as time changes. Thus the expression above coincides with the standard matrix elements for the computation of quarkonia states [48]. For the production in the medium-brick, the situation is more complicated, since the interaction with the QGP fields changes the potential during the time extent of the medium; furthermore, when the $Q-\bar{Q}$ leaves the QGP-brick, the potential changes abruptly back to the vacuum expression. If all the thermal effects on the $Q-\bar{Q}$ system can be expressed in terms of a medium-modified potential, the complicated dynamics of the production process in this set up can be addressed by solving the time dependent Schrödinger equation, eq. (3.7) once the explicit form of the potential is known.

\subsection{Quarkonia Suppression in a QGP-Brick}

Since after the particle leaves the brick the yield of the different states remains constant, in order to determine the production of quarkonia in the system it is enough to compute the evolution of the hard density matrix up to the time $t$ when the medium disappears. Following the previous discussion, the yield of a given state is obtained by (numerically) solving the time dependent Schrödiger equation, eq (3.7), with the in-medium potential, eq. (2.2) and eq. (2.1), starting at $\tilde{t}=0$ with its vacuum wave functions and computing the value of the evolved wave function at the origin at time $t$. From this solution I determine the yield suppression by the medium, which I express, as customary in heavy ion physics, as the ratio of the yields after the QGP-brick to the one in vacuum

$$
R_{S}=\frac{Y_{S}\left(\mu_{D}\right)}{Y_{S}\left(\mu_{D}=0\right)} .
$$

In the left panel of fig. (4) I show the suppression of the S-wave ground state (1S) for different values of $\mu_{D}$ as a function of the time duration of the box in units of the inverse period of the ground state wave function $\mathcal{T}=2 \pi / E_{b}$, which I will identify as the formation time. For very small brick lifetimes, less than one period, the suppression is relatively modest for all values of $\mu_{D}$. At later times and as long as the in medium potential supports a bounded ground state, the yield suppression saturates to a constant value, which is determined by the value of the in-medium wave function at the origin and the overlap of those wave functions with the vacuum ground state. As $\mu_{D}$ grows, the late time suppression also grows, since the in-medium states becomes wider as they approach threshold, reducing both the value of their wave function at zero and the overlap with the vacuum wave function. When $\mu_{D}$ is so large that the ground states disappears (not shown) the late time yield tends to zero.

I have also performed a similar analysis for the suppression of the next vacuum state, the $2 \mathrm{~S}$ level. In the right panel of Fig (4) I show the double ratio of the suppression factor of 

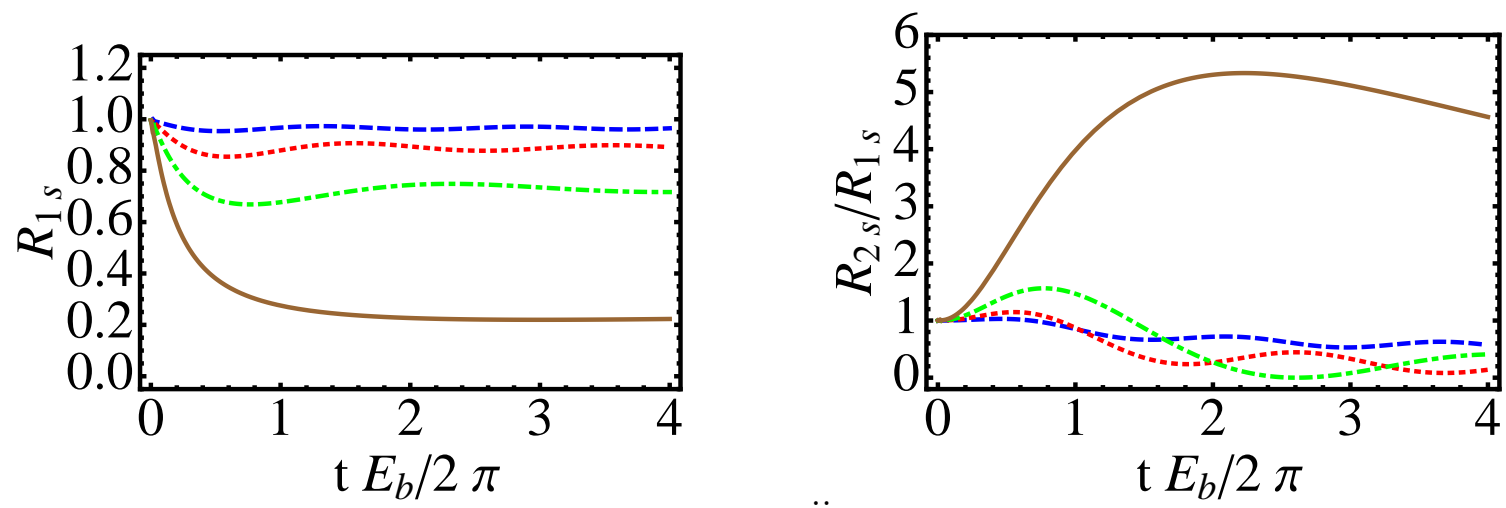

Figure 4. Left: Yield suppression of the ground state level as a function of the lifetime of the QGP-brick, $\mathrm{t}$, in units of the vacuum ground state period $\mathcal{T}=2 \pi / E_{b}$. Right: Double ratio of the suppression factors of $2 \mathrm{~S}$ and $1 \mathrm{~S}$ states as function of the lifetime of the QGP-brick. Both calculations are for the real potential eq (2.1) with $2 \mu_{D} / \alpha M=0.15,0.3,0.5,0.9$ for the dashed, dotted, dash dotted and solid lines respectively.

the $2 \mathrm{~S}$ state to the $1 \mathrm{~S}$ estate. Note that for only one of the cases considered, $\mu_{D}=0.15 \alpha M / 2$ (dashed line in fig. (4) ) the in-medium potential supports $2 \mathrm{~S}$ bound level and the medium modifications are small. As the temperature rises, a modulation in the double yield ratio appears. The physical origin of this is simple and it is due to the non-zero overlap of the vacuum $2 \mathrm{~S}$ state with the in-medium ground state, which for this potential survives for all the shown values of $\mu_{D}$. The period of oscillation is given by the in-medium ground state energy and the amplitude is controlled by the interference between the in-medium continuous states and the ground state, which disappears at later times since the former states are delocalized. Quite remarkably, this double ratio can be larger than 1, showing a relative enhancement of $2 \mathrm{~S}$ to $1 \mathrm{~S}$ states. This is particularly dramatic in the highest temperature case, for which the bound states are loosely bound. This behavior continues at even higher temperatures, when all states are dissolved and it is due to the fact that the vacuum $2 \mathrm{~S}$ state is wider than the ground state and has a larger overlap with the in-medium continuum. However, I emphasize that despite the relative enhancement, in this situation both the $1 \mathrm{~S}$ and $2 \mathrm{~S}$ states are strongly suppressed as compared to the vacuum.

The introduction of an imaginary part to the potential leads to some distinct features in the suppression pattern of the QGP brick. As shown in the left panel of fig. (5), for the same values of $\mu_{D}$, the complex potential, eq. (2.2) is much more effective in suppressing quarkonia. Contrary to fig. (4), for this potential the late time suppression does not saturate to a constant, but continuously increases due to the finite width of the in-medium states. In fact, at these late times, the dynamics of the in-medium ground state is dominated by this absorption. This is demonstrated in the right panel of fig. (5), where I show the ratio of the numerically computed suppression divided by the expected absorption rate due to the width $\Gamma$ of the in-medium ground state for the values of $\mu_{D}$ for which it survives. As shown by the plot, after the formation time of the meson the absorption is indeed given by the in-medium width up to an overall shift, which is due to the early time suppression. 

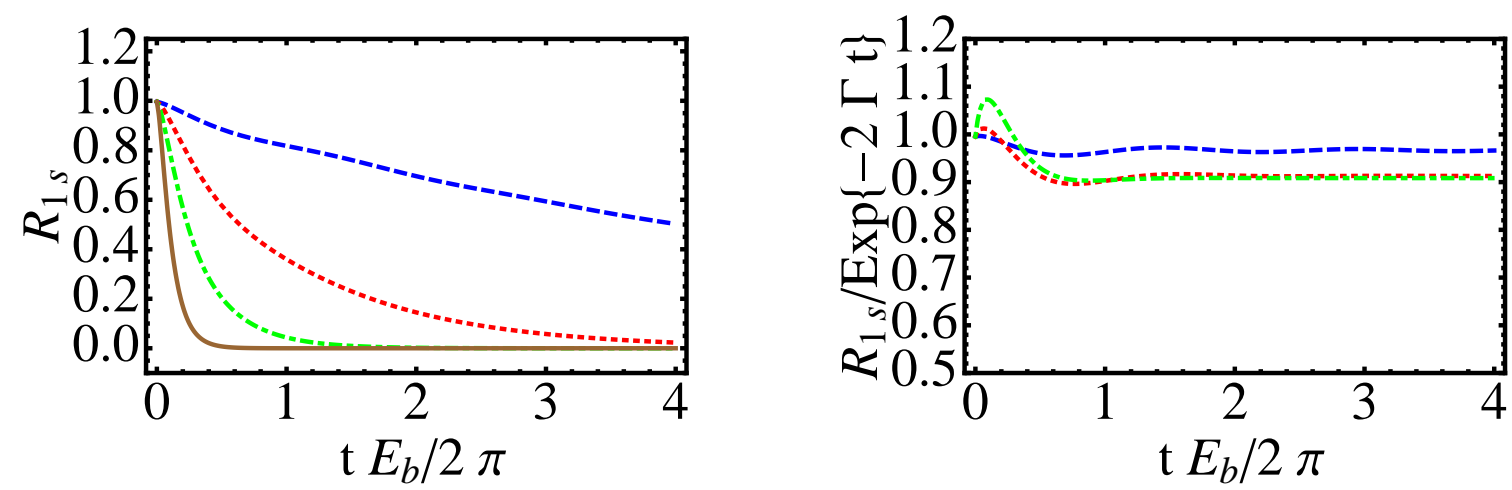

Figure 5. Left: Yield suppression of the ground state level as a function of the lifetime of the QGPbrick, $\mathrm{t}$, in units of the vacuum ground state period $\mathcal{T}=2 \pi / E_{b}$ for the complex potential eq $(2.2)$ for different values of $\mu_{D}, 2 \mu_{D} / \alpha M=0.15,0.3,0.5,0.9$. Right: Comparison of the suppression factor to the attenuation of the yield expected from the imaginary part of the lowest in-medium mode for the previous values of $\mu_{D}$ which support an in-medium state.
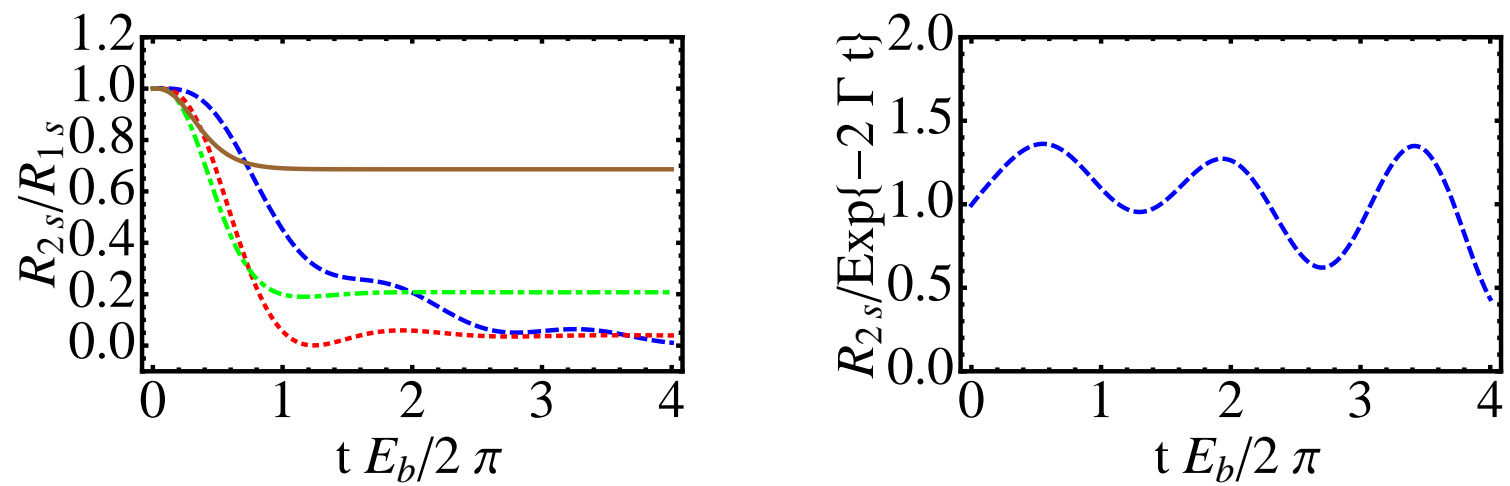

Figure 6. Left: Double ratio of the suppression factors of the $2 \mathrm{~S}$ and $1 \mathrm{~S}$ states as a function of the lifetime of the QGP-brick, $t$, in units of the vacuum ground state period $\mathcal{T}=2 \pi / E_{b}$ for the complex potential eq (2.2) for different values of $\mu_{D}, 2 \mu_{D} / \alpha M=0.15,0.3,0.5,0.9$. Right: Comparison of the suppression factor to the attenuation of the yield expected from the imaginary part of the $2 \mathrm{~S}$ in-medium mode for the only value of $\mu_{D}$ which supports a bound $2 \mathrm{~S}$ state.

In the left panel of fig. (6) I show the double ratio of suppression of the $2 \mathrm{~S}$ to $1 \mathrm{~S}$ state for this imaginary potential. For the three highest values of $\mu_{D}$ shown, for which the potential does not support a $1 \mathrm{~S}$ level, the late time ratio is constant, which means that both the $2 \mathrm{~S}$ and $1 \mathrm{~S}$ yields fall at the same rate. Similarly to the real potential case, this is due to the fact that when the in-medium $2 \mathrm{~S}$ level is absent, the production is dominated by the overlap with the in-medium 1S. Once again, the typical time scale to reach to this asymptotic behavior is given by the ground state period. As in the real potential case, the asymptotic value grows with $\mu_{D}$. Even though the double ratio does not go above one for any of the shown values, I have checked that further increasing $\mu_{D}$ leads to an enhancement over the vacuum, as in the previous case, but such that each individual yield is strongly suppressed.

The effect of the observed level overlap is also present for colder media, in which a $2 \mathrm{~S}$ 
level exist. In the right panel of fig. (6) I compare the numerically computed suppression factor to the absorption rate due to the imaginary part on the in-medium level. Contrary to the ground state, the rate is not saturated by the in-medium level, and there are large oscillations around this decay. The period of those agrees, in fact, with the real part of the in-medium 1S level. Furthermore, since the imaginary part of the 1S level is smaller than the $2 \mathrm{~S}$ one, the ground state contribution grows at late times.

In summary, by comparing the suppression patterns of a medium-brick characterized by a real quarkonia potential to that of a potential with an imaginary part with the same screening parameter $\mu_{D}$ I have shown that the non-unitarity effects introduced by the complex potential are very efficient in suppressing quarkonia. Even a relatively small imaginary part has dramatic consequences in the late time suppression of quarkonia, specially for long lived medium-bricks. On the contrary, for short lived bricks, with a lifetime less than the formation time, the differences between these two models of the medium are not so dramatic. Additionally, I have shown that even though the ground state suppression pattern is easy to understand in terms of in-medium bound states, the suppression of excited states, such as $2 \mathrm{~S}$-wave levels, have a much richer structure which arises not only from the contribution of the corresponding $2 \mathrm{~S}$ in-medium level, but also from the ground state, which in some cases become dominant.

\section{Quarkonia Suppression in an Expanding Medium}

The analysis of the static brick in the previous section, in which the medium parameters are fixed during the medium lifetime, shows that the in-medium suppression patterns develop in a characteristic time of the order of the ground state period. A simple estimate of these times for chamonium and bottomonium bound states yields typical formation times $\mathcal{T}$ of few $\mathrm{fm}$, which having in mind any heavy ion physics applications, are large, comparable to the whole fireball lifetime. Thus, in this section I will extend the analysis to an "expanding" brick, for which I mean a spatially infinite homogeneous medium whose properties change continuously with time, till the medium completely disappears at a given time. I will assume that the internal processes in the medium are sufficiently fast to keep the medium thermalized during the expansion. Motivated by the dynamics of boost invariant plasmas, a common approximation in describing hydrodynamic evolution in heavy ion collisions, I will assume a time dependence of $\mu_{D}$ given by

$$
\mu_{D}(t)=\mu_{D 0}\left(\frac{\tau_{0}}{t+\tau_{0}}\right)^{1 / 3},
$$

with $\mu_{0}$ the initial value of the Debye screening length and $\tau_{0}$ a thermalization time parameter which I will take to be $\tau_{0}=0.4 \mathrm{fm}$. To mimic the deconfinement transition, I demand that the brick lifetime coincides with the time in with $\mu_{D}=175 \mathrm{MeV}$, the phase transition temperature, where I am assuming, as already mention in section 2 , that in the vicinity of $T_{c}, \mu_{D} \approx T$. In a real expansion, the hadronic medium does not cease to exist after the system reaches $T_{c}$. However, after this time the in-medium modifications of quarkonia are 

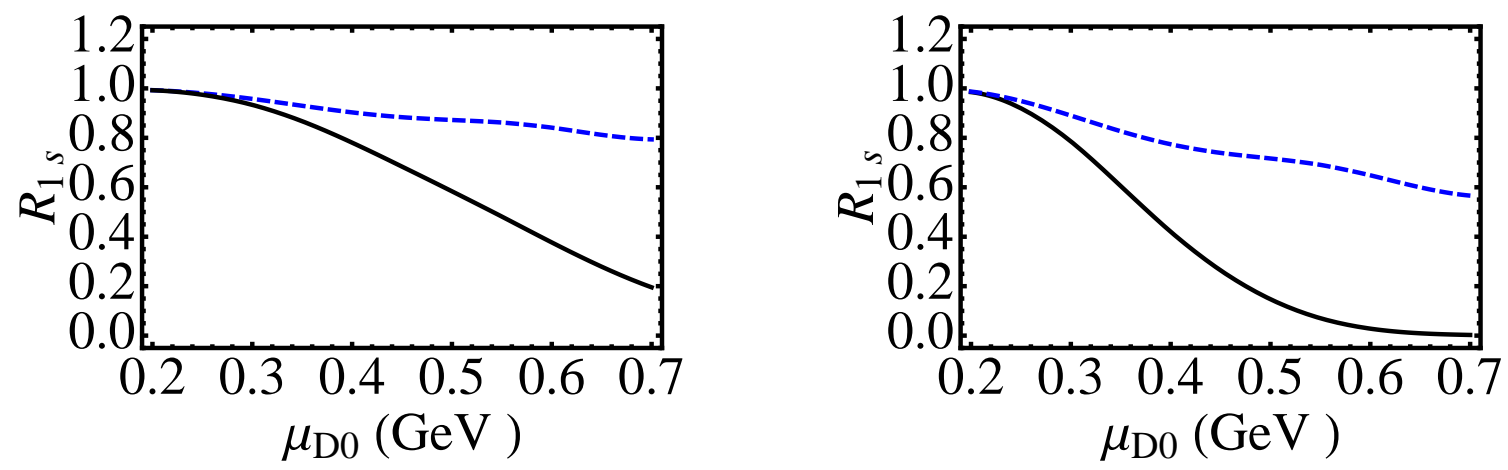

Figure 7. Suppression factor of the 1S state in a finite lifetime expanding medium as a function of the initial value of the $\mu_{D}$ parameter for both the real (dashed) and complex (solid) potentials. The left and right panels correspond to $\Upsilon$ and $J / \psi$ suppressions respectively.

much smaller, since the medium is confined. In this simplified approach I am neglecting the interaction of quarkonia states with the dense hadronic medium after the transition.

To study the effect of the expanding and deconfined medium into the different quarkonia states, I need to set physical values for the wave function parameters. Since given the many assumptions of this exploratory work I will not be able to make direct contact with experimental data, I will restrict myself to the coulombic wave functions used in the previous analysis without questioning how approximate they are to those of a realistic potential. To fix the scale, I will equate the r.m.s of the coulombic wave function, $r_{r m s}=<r^{2}>^{1 / 2}$, to the one of wave function computed with a realistic vacuum potential [32, 38]. Using $r_{r m s}=0.5,0.3 \mathrm{fm}$ for $J / \psi$ and $\Upsilon$ respectively, the effective $\alpha$ is different for charm and bottom bound states, $\alpha=1,0.5$ respectively. This value also sets the binding energies of both ground states, $E_{b}=0.32,0.26 \mathrm{GeV}$ for $J / \psi$ and $\Upsilon$. These binding energies are low as compared to the binding energies of vacuum $J / \psi$ and $\Upsilon$ states $E_{b} \approx 0.7,1 \mathrm{GeV}$, respectively; however, they are close to the in-medium binding energies right above $T_{C}$ extracted from potential model description of lattice heavy quark current-current correlators $[34,35]$, which is the relevant scale for describing the decorrelation among different inmedium states. Nevertheless, because of this mismatch, all the time scale estimates in this work should be taken as indicative.

I now repeat the analysis in the previous section with the time dependent potential arising from the use of eq. (4.1) in eq. (3.7) and eq. (2.2). As in the static case, I evolve the vacuum wave functions with the Schrödinger equation, eq. (3.7), up to the final time, defined by $\mu_{D}=T_{c}$. In fig. (7) I show the suppression of the $\Upsilon$ (left) and $J / \psi$ (right) states both for real (dashed) and complex potentials (solid), as a function of the initial screening length $\mu_{D 0}$. As expected from the analysis of the static medium, the complex potential is much more effective in suppressing quarkonia states than the real one. While the real potential leads to a moderate suppression both for $J / \psi$ and $\Upsilon$, the complex potential leads to a larger suppression with a stronger dependence on the initial $\mu_{D}$ value. Note that with the current choice of parameters, for the most part of the evolution the value of $\mu_{D}$ at any time is smaller than the dissociation value, inferred from fig. (2). The suppression pattern 

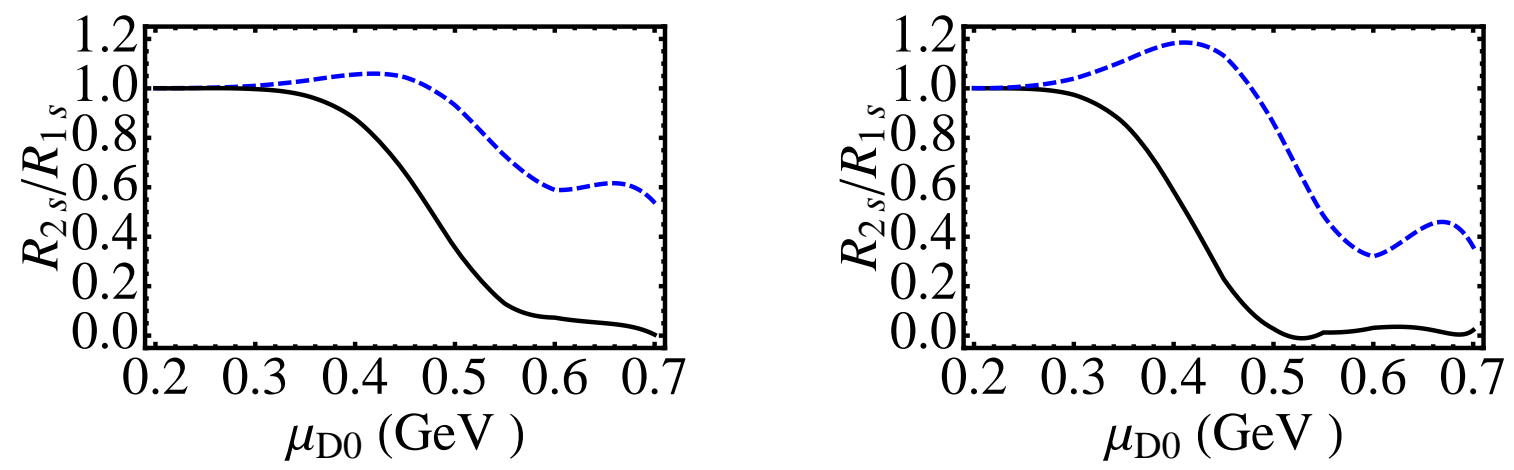

Figure 8. Double ratio of the suppression factors of the $2 \mathrm{~S}$ to $1 \mathrm{~S}$ states in a finite lifetime expanding medium as a function of the initial value of the $\mu_{D}$ parameter for both the real (dashed) and complex (solid) potentials. The left and right panels correspond to $\Upsilon$ and $J / \psi$ suppressions respectively.

observed is, in fact, a consequence of the transient behavior appearing at short times in the fixed $\mu_{D}$ calculations of the previous section, since for $\mu_{D 0}<0.5 \mathrm{GeV}$ the lifetime of the medium is smaller than $\mathcal{T}$.

In fig. (8) I show the double ratio of the suppression factors of $2 S$ and $1 S$ states. Contrary to the fixed temperature calculation, neither for the $\Upsilon$ nor $J / \psi$ case the double ratio is significantly larger than one, despite of the fact that, at least for $J / \psi$, the highest values of $\mu_{D}$ are sufficiently large to expect an enhancement. The reason for this lack of enhancement is that even for the hottest initial medium, the fast evolution at early times makes $\mu_{D}$ to drop significantly within the transient time, $\mathcal{T}$. Thus, the final yield is dominated by low values of $\mu_{D}$ for which, within the current parameter set, the $1 \mathrm{~S}$ states are bound. Note, however, that because of my simplified choice of in-medium potentials, quarkonia states survive till large values of the $\mu_{D}$, in contrast with other more realistic potential model calculations [33, 34]. Nevertheless, this calculation shows the insensitivity of the suppression pattern to the very early stages of the evolution, where the temperature is the highest, as a consequence of the finite formation time of the different quarkonia states.

\section{Quarkonia Suppression in a non-Homogenous Expanding Medium}

Since there is a non-trivial relation between the static brick studied in section (3.2) and the expanding case of the previous section, I will now investigate the effect of a non-homogenous temperature profile in the final quarkonia production rate after a finite time in the hot medium. Having in mind ultra relativistic heavy ion collision applications, I will consider a medium of finite spacial extent with varying initial $\mu_{D 0}$ which expands at a (local) rate given by eq. (4.1). In this study, the initial parameter $\mu_{D 0}$ becomes position dependent; however I will assume that the typical size of in-medium bound states is small as compared to the typical variation of the $\mu$-profile of the medium and neglect the spatial variation of the in-medium hamiltonian.

As a simplified model for the medium formed after the collision of the two heavy nuclei I will use a hard spheres model with a strong longitudinal expansion but no transverse 

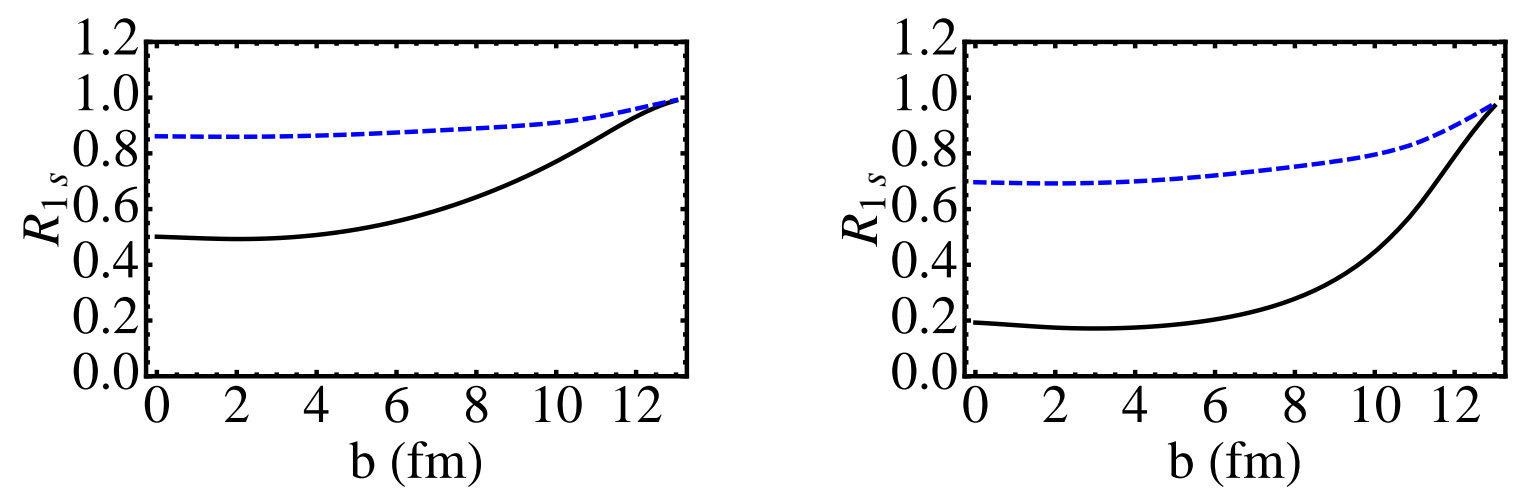

Figure 9. Suppression factor of the 1S state for the spherical nuclei collisions as a function of impact parameter $b$ for both the real (dashed) and complex (solid) potentials. The left and right panels correspond to $\Upsilon$ and $J / \psi$ suppressions respectively.

dynamics. Right after the collision, there is a non-vanishing temperature profile inside the overlap region of the two spheres. At any impact parameter $b$, this profile is given by

$$
\mu_{D}=\mathcal{C}\left(T_{A}((x-b / 2), y)+T_{A}((x+b / 2), y)\right)
$$

with $T_{A}(x, y)=\int \rho(x, y, z) d z$ the thickness function of the nuclei of density $\rho$ and $\mathcal{C}$ a proportionality constant. For this study, I will set $\mathcal{C}$ by demanding that in central collisions $(b=0)$ the highest value of $\mu_{D}=0.7 \mathrm{GeV}$. This procedure assigns to every point in the transverse plane of the overlap region a non-vanishing value of $\mu_{D}$ which decreases as the point is further away from he center of that region. As in the previous sections I will assume that if $\mu_{D}<0.175 \mathrm{GeV}$ there is not a substantial modification of the in-medium potential and for all those points of the transverse plane for which eq. (5.1) yields values smaller than $T_{c}$ I set $\mu_{D}=0$. For all the other points, $\mu_{D}$ decreases with time as dictated by eq. (5.1).

Having determined the $\mu_{D}$ profile, I also need to specify the distribution of production points of the $Q-\bar{Q}$ pairs. Without specifying the microscopic mechanism that leads to the creation of pairs in singlet state, I will assume that the probability of production scales with the number of binary collisions

$$
\mathcal{P} \propto T_{A A}(x, y ; b)
$$

with $T_{A A}(x, y ; b)=T_{A}(x-b / 2, y) T_{A}(x+b / 2, y)$ defined as usual. I will also assume that the $Q-\bar{Q}$ pair is produced mostly at rest and leave the momentum dependence of the suppression for a future study. Thus, the effective hamiltonian governing the soft dynamics of the pair is controlled by the local temperature at the production point and its subsequent time evolution. Averaging over the overlap region I obtain the yield of the different states associated to this simplified model of nuclear collisions.

In fig. (9) I show the suppression factor of $1 \mathrm{~S}$ states for the spherical nuclei collisions obtained by comparing the in-medium production to the production obtained with the vacuum potential. In the left (right) panel I show the production rate for the $\Upsilon 1 \mathrm{~S}(J / \psi)$ for the two model potentials as a function of the impact parameter. As inferred from the 

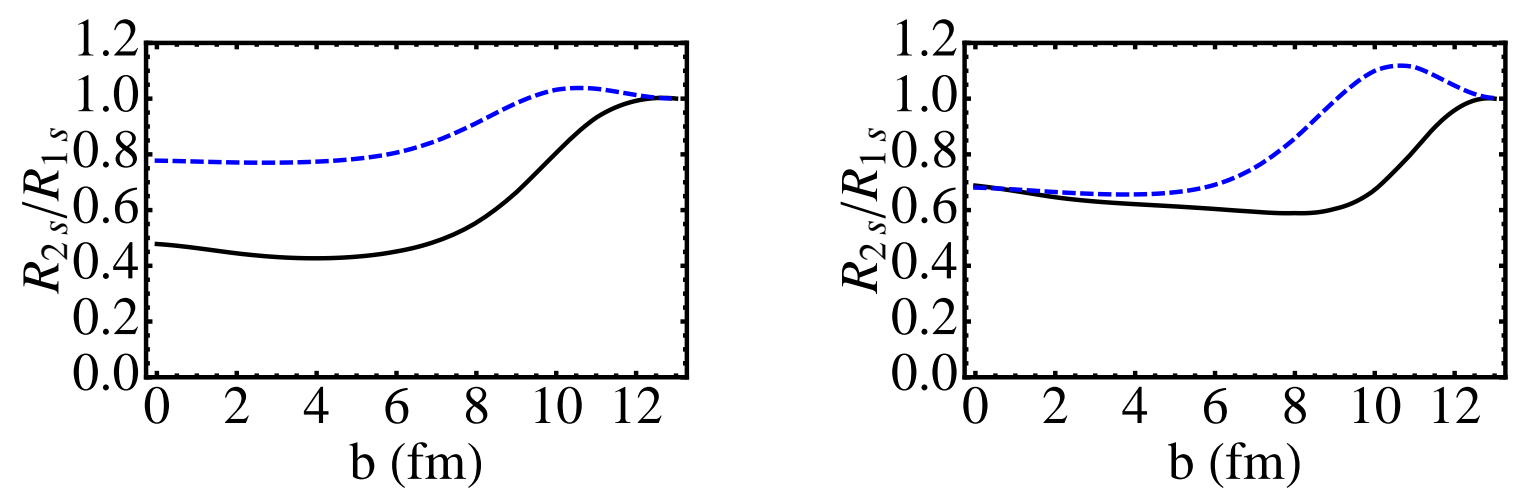

Figure 10. Double ratio of the suppression factors of the $2 \mathrm{~S}$ to the $1 \mathrm{~S}$ state for the spherical nuclei collisions as a function of impact parameter $b$ for both the real (dashed) and complex (solid) potentials. The left and right panels correspond to $\Upsilon$ and $J / \psi$ suppressions respectively.

fixed $\mu_{D}$ studies, the complex potential is much more effective in suppressing both quarkonia states while the real potential leads to a modest suppression in both cases. As expected, since $J / \psi$ states melt at a lower temperature than the $\Upsilon$ ground state, the suppression is larger for the former than from the latter. For the particular parameter set in this study, the in-medium potential supports bound $1 \mathrm{~S} \Upsilon$ and $J / \psi$ states in most of the evolution and is only able to dissolve the $J / \psi$ for the hottest part of the most central collisions. However, and in spite of the different dissociation temperatures, the nuclear absorption is approximately constant until similar values of the impact parameter, $b \sim 8 \mathrm{fm}$. This fact is a consequence of the long formation time of the in-medium bound states, since increasing $b$ leads to a shorter lifetime of the fireball. For the collision model I have considered, $b=8$ $\mathrm{fm}$ corresponds to the the largest impact parameter for which the fireball lifetime is longer than the quarkonia formation time.

In fig.. (10) I show the double ratio of the suppression factors of the $2 \mathrm{~S}$ to the $1 \mathrm{~S}$ levels for the $\Upsilon$ and $J / \Psi$ families both for real and complex potentials. As in all the previous cases, the complex potential is more effective in suppressing $2 \mathrm{~S}$ quarkonia states. As for the ground state case, the $b$ dependence of the suppression is relatively featureless. For $b<6 \mathrm{fm}$, the double ratio decreases slightly as $b$ increases. This is a consequence of the hotter fireball in more central collisions and the observation made in section (3.2) that as the in-medium ground states moves towards threshold, there is an enhancement in the relative production of $2 \mathrm{~S}$ to $1 \mathrm{~S}$ states. However, contrary to the fixed temperature cases in section (3.2), this double ratio does not significantly cross unity for any $b$. As in the expanding case, this is a consequence of the particular choice of parameters in this articles, since for most of the evolution the potentials I have considered support a bound level. As already mentioned, this feature is in contrast with lattice studies of charm current-current correlators which indicate the dissolution of $J / \psi$ states soon after $T_{c}$. Finally, as for the ground state case, the double ratio rises for $b \sim 8 \mathrm{fm}$, showing once again that this non-trivial behavior is due to the lifetime of the fireball as compared to the meson formation time. 


\section{Discussion}

Following the potential model approach to in-medium heavy quarkonia physics, the main assumption of this work is that all relevant interactions of a heavy quark pair with the QGP can be recast in a temperature dependent potential. In particular, I have assumed that all level dissociation effects by plasma interactions with the pair can be encoded in the potential without demanding additional processes. If the medium is described by a real potential, the interactions lead to a reorganization of levels which are conserved in the medium. On the contrary, complex potentials naturally include dissociation processes, such as the Landau damping (see [6] for a discussion on the different dissociation process), in the imaginary par of the potential. With this approach I have calculated the suppression pattern of different quarkonia states by a finite lifetime medium interacting with a heavy $Q-\bar{Q}$ pair produced by some hard process in a singlet state.

Potential models have the advantage that, once the correct singlet potential is obtained, they can be directly used to describe both the late time soft matrix element needed in quarkonia production as well as the current-current correlators extracted by lattice QCD calculations. In addition, since this approach is able to treat simultaneously all the states of the $Q-\bar{Q}$ system, it allows for a complete determination of the yield suppression taking into account the overlap between different vacuum and in-medium wave functions. Unfortunately, there is currently no consensus on the functional form of the potential which best describes lattice data in the vicinity of $T_{c}$. As a consequence, in this article I have used two simple potentials which lead to the sequential disappearance of the different bound states, without any attempt to adjust to the lattice data. This oversimplified approach is sufficient to draw the main conclusions of this study and I leave a more refined treatment of the potential for future work.

One of the main observations of this work is that, while the productions of $1 \mathrm{~S}$ levels is dominated by the properties of the in-medium ground state, the production of higher excited states, such as the $2 \mathrm{~S}$ ones, is more complicated. As I have shown in section 3.2 these states are also influenced by the in-medium ground state in addition to the in-medium level with the same quantum numbers. Thus, to properly describe the suppression of quarkonia excited states it is not enough to understand the in-medium properties of these states but also the mixing of states which occurs in the medium, since vacuum and in-medium wave functions have non-trivial overlap.

This interplay among different states for the production of $2 \mathrm{~S}$ states also leads to some unexpected features in their suppression pattern. In the fixed temperature calculations in section 3.2 I have shown that if the in-medium ground state is totally dissolved or if it is sufficiently close to threshold there is a strong enhancement in the relative production of $2 \mathrm{~S}$ to $1 \mathrm{~S}$ states. This enhancement is generic, independent of the particular details of the in-medium potential, since it is only a consequence of the fact that the lesser bound the in-medium levels are the wider they become, increasing their overlap with vacuum excited states. While the potential approach is more suited for bottomonium states, this feature of the potential model calculation goes along the trends observed in preliminary $\psi(1 \mathrm{~S})$ production data by CMS [17], which indicate a strong enhancement of the double ratio of 
$\psi(1 S)$ to $J / \psi R_{A A}$ at moderate $p_{T}$. However, a more detailed study is needed before a direct comparison with data can be made.

I have also studied the effect of an expanding medium in quarkonia production. Under the assumption that the expansion rate is sufficiently slow as compared to the internal processes in the plasma, I have used this potential model approach to address the dynamics of the system in a pre-asymptotic stage (see $[49,50]$ for related studies) and I have identified the natural scale for the formation of in-medium states, the ground state period. While for this exploratory study I have used low binding energies for the different vacuum quarkonia states, these formation time effects are important and make the quarkonia production mostly insensitive to the hottest part of the medium evolution. This observation suggests that the suppression pattern of quarkonia in nuclear collisions must have a modest dependence on the colliding energy since the higher temperatures reached in energetic collisions are rapidly relaxed due to the strong longitudinal expansion. Remarkably, this is precisely the trend observed in comparing suppression data from SPS to the LHC [11-16]. However, a much more detailed analysis than the one in this work must be performed before we can determine the relevance of these effects for the observed patterns in suppression data.

These formation time effects have also a important effect in the pattern of suppression of different quarkonia levels. While for a static medium there is an enhancement of the relative abundance of excited to ground states whenever the in-medium ground state is dissolved or close to threshold, the situation in an expanding medium is more complicated. In fact, even if at the early stages of the system evolution the medium is sufficiently hot to dissolve the ground state of a given quarkonia family, if for most of the evolution the ground state is present, the enhancement in the double ratio of $2 \mathrm{~S}$ to $1 \mathrm{~S}$ states observed in the static medium will not have time to develop. Once again, this observation has profound consequences for quarkonia physics at the LHC: If the anomalous suppression pattern observed in preliminary CMS data can be attributed to this mixing effect between states below and above the transitions, the relative enhancement of $\psi(2 S)$ production indicates that $J / \psi$ mets for the most part of the hot medium evolution at the LHC, which, in turn, points towards a low melting temperature of in-medium quarkonia

In summary, the potential model calculation I have explored in this paper is a simple and powerful method to describe the suppression patter of quarkonia in heavy ion collisions. The analysis in this work has been oversimplified not only because of the simplified potentials that I have used, but also for the simplified treatment of quarkonia production which, in particular, neglects octect contribution, the lack of energy loss of the pair, the interaction of more than one pair created in the collision, initial state effects, etc. All these processes must be address before drawing any firm conclusion about quarkonia suppression in data. Nevertheless, some of the features I have discussed, such as the complicated interplay among vacuum and medium levels are completely generic of this approach, independently of the details of the potential. 


\section{Acknowledgments}

I thank F. Arleo, E. G. Ferreiro, A. Palacios and J. Soto for useful discussions. I am supported by a Ramón y Cajal fellowship. I also acknowledge financial support by the research grants FPA2010-20807, 2009SGR502 and by the Consolider CPAN project.

\section{References}

[1] PHENIX Collaboration Collaboration, K. Adcox et al., Formation of dense partonic matter in relativistic nucleus-nucleus collisions at RHIC: Experimental evaluation by the PHENIX collaboration, Nucl.Phys. A757 (2005) 184-283, [nucl-ex/0410003].

[2] STAR Collaboration Collaboration, J. Adams et al., Experimental and theoretical challenges in the search for the quark gluon plasma: The STAR Collaboration's critical assessment of the evidence from RHIC collisions, Nucl.Phys. A757 (2005) 102-183, [nucl-ex/0501009].

[3] B. Muller, J. Schukraft, and B. Wyslouch, First Results from $\mathrm{Pb}+\mathrm{Pb}$ collisions at the $\mathrm{LHC}$, arXiv: 1202.3233.

[4] T. Matsui and H. Satz, J/psi Suppression by Quark-Gluon Plasma Formation, Phys.Lett. B178 (1986) 416.

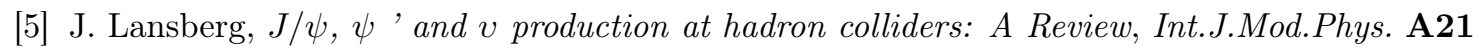
(2006) 3857-3916, [hep-ph/0602091].

[6] R. Rapp, D. Blaschke, and P. Crochet, Charmonium and bottomonium production in heavy-ion collisions, Prog.Part.Nucl.Phys. 65 (2010) 209-266, [arXiv:0807.2470].

[7] P. Braun-Munzinger and J. Stachel, (Non)thermal aspects of charmonium production and a new look at J / psi suppression, Phys.Lett. B490 (2000) 196-202, [nucl-th/0007059].

[8] R. L. Thews, M. Schroedter, and J. Rafelski, Enhanced J / psi production in deconfined quark matter, Phys.Rev. C63 (2001) 054905, [hep-ph/0007323].

[9] M. I. Gorenstein, A. Kostyuk, H. Stoecker, and W. Greiner, Statistical coalescence model with exact charm conservation, Phys.Lett. B509 (2001) 277-282, [hep-ph/0010148].

[10] L. Grandchamp and R. Rapp, Thermal versus direct J / Psi production in ultrarelativistic heavy ion collisions, Phys.Lett. B523 (2001) 60-66, [hep-ph/0103124].

[11] NA50 Collaboration Collaboration, M. Gonin et al., Anomalous J / psi suppression in Pb $+\mathrm{Pb}$ collisions at 158-A-GeV/c, Nucl.Phys. A610 (1996) 404C-417C.

[12] NA50 Collaboration Collaboration, M. Abreu et al., Anomalous J / psi suppression in Pb - Pb interactions at $158 \mathrm{GeV/c}$ per nucleon, Phys.Lett. B410 (1997) 337-343.

[13] PHENIX Collaboration Collaboration, A. Adare et al., J/psi Production vs Centrality, Transverse Momentum, and Rapidity in Au+Au Collisions at $s(N N)^{* *}(1 / 2)=200-G e V$, Phys.Rev.Lett. 98 (2007) 232301, [nucl-ex/0611020].

[14] Atlas Collaboration Collaboration, G. Aad et al., Measurement of the centrality dependence of $J / \psi$ yields and observation of $Z$ production in lead-lead collisions with the ATLAS detector at the LHC, Phys.Lett. B697 (2011) 294-312, [arXiv:1012.5419].

[15] ALICE Collaboration Collaboration, B. Abelev et al., J/psi production at low transverse momentum in $\mathrm{Pb}-\mathrm{Pb}$ collisions at sqrt $(s N N)=2.76 \mathrm{TeV}$, arXiv:1202.1383. 
[16] CMS Collaboration Collaboration, S. Chatrchyan et al., Suppression of non-prompt J/psi, prompt $\mathrm{J} / \mathrm{psi}$, and $\mathrm{Y}(1 \mathrm{~S})$ in PbPb collisions at sqrt(sNN) =2.76 TeV, JHEP 1205 (2012) 063, [arXiv: 1201.5069].

[17] CMS Collaboration Collaboration, T. Dhams, talk given at the 5th international conference on hard and electromagnetic probes of high-energy nuclear collisions (hp2012)., .

[18] R. Reed, Measuring the Upsilon Nuclear Modification Factor at STAR, J.Phys.G G38 (2011) 124185, [arXiv: 1109.3891].

[19] M. Asakawa and T. Hatsuda, J / psi and eta(c) in the deconfined plasma from lattice QCD, Phys.Rev.Lett. 92 (2004) 012001, [hep-lat/0308034].

[20] S. Datta, F. Karsch, P. Petreczky, and I. Wetzorke, Behavior of charmonium systems after deconfinement, Phys.Rev. D69 (2004) 094507, [hep-lat/0312037].

[21] R. Morrin, A. O Cais, M. Oktay, M. Peardon, J. Skullerud, et al., Charmonium spectral functions in $N_{f}=2$ QCD, PoS LAT2005 (2006) 176, [hep-lat/0509115].

[22] A. Jakovac, P. Petreczky, K. Petrov, and A. Velytsky, Quarkonium correlators and spectral functions at zero and finite temperature, Phys.Rev. D75 (2007) 014506, [hep-lat/0611017].

[23] G. Aarts, C. Allton, M. B. Oktay, M. Peardon, and J.-I. Skullerud, Charmonium at high temperature in two-flavor QCD, Phys.Rev. D76 (2007) 094513, [arXiv:0705.2198].

[24] G. Aarts, C. Allton, S. Kim, M. Lombardo, M. Oktay, et al., What happens to the Upsilon and et $_{b}$ in the quark-gluon plasma? Bottomonium spectral functions from lattice $Q C D$, JHEP 1111 (2011) 103, [arXiv:1109.4496].

[25] H.-T. Ding, A. Francis, O. Kaczmarek, F. Karsch, H. Satz, et al., Charmonium properties in hot quenched lattice QCD, arXiv:1204.4945.

[26] F. Karsch, M. Mehr, and H. Satz, Color Screening and Deconfinement for Bound States of Heavy Quarks, Z.Phys. C37 (1988) 617.

[27] S. Digal, P. Petreczky, and H. Satz, Quarkonium feed down and sequential suppression, Phys.Rev. D64 (2001) 094015, [hep-ph/0106017].

[28] E. V. Shuryak and I. Zahed, Towards a theory of binary bound states in the quark gluon plasma, Phys.Rev. D70 (2004) 054507, [hep-ph/0403127].

[29] C.-Y. Wong, Heavy quarkonia in quark-gluon plasma, Phys.Rev. C72 (2005) 034906, [hep-ph/0408020].

[30] W. Alberico, A. Beraudo, A. De Pace, and A. Molinari, Heavy quark bound states above T(c), Phys.Rev. D72 (2005) 114011, [hep-ph/0507084].

[31] M. Mannarelli and R. Rapp, Hadronic modes and quark properties in the quark-gluon plasma, Phys.Rev. C72 (2005) 064905, [hep-ph/0505080].

[32] A. Mocsy and P. Petreczky, Quarkonia correlators above deconfinement, Phys.Rev. D73 (2006) 074007, [hep-ph/0512156].

[33] A. Mocsy and P. Petreczky, Can quarkonia survive deconfinement?, Phys.Rev. D77 (2008) 014501, [arXiv:0705.2559].

[34] A. Mocsy and P. Petreczky, Color screening melts quarkonium, Phys.Rev.Lett. 99 (2007) 211602, [arXiv:0706.2183]. 
[35] D. Cabrera and R. Rapp, T-Matrix Approach to Quarkonium Correlation Functions in the QGP, Phys.Rev. D76 (2007) 114506, [hep-ph/0611134].

[36] M. Laine, A Resummed perturbative estimate for the quarkonium spectral function in hot QCD, JHEP 0705 (2007) 028, [arXiv:0704.1720].

[37] O. Kaczmarek and F. Zantow, Static quark anti-quark interactions in zero and finite temperature QCD. I. Heavy quark free energies, running coupling and quarkonium binding, Phys.Rev. D71 (2005) 114510, [hep-lat/0503017].

[38] E. Eichten, K. Gottfried, T. Kinoshita, K. Lane, and T.-M. Yan, Charmonium: Comparison with Experiment, Phys.Rev. D21 (1980) 203.

[39] M. Laine, O. Philipsen, P. Romatschke, and M. Tassler, Real-time static potential in hot QCD, JHEP 0703 (2007) 054, [hep-ph/0611300].

[40] A. Beraudo, J.-P. Blaizot, and C. Ratti, Real and imaginary-time $Q$ anti- $Q$ correlators in a thermal medium, Nucl.Phys. A806 (2008) 312-338, [arXiv:0712.4394].

[41] M. A. Escobedo and J. Soto, Non-relativistic bound states at finite temperature (I): The Hydrogen atom, Phys.Rev. A78 (2008) 032520, [arXiv:0804.0691].

[42] N. Brambilla, J. Ghiglieri, A. Vairo, and P. Petreczky, Static quark-antiquark pairs at finite temperature, Phys.Rev. D78 (2008) 014017, [arXiv:0804.0993].

[43] N. Brambilla, M. A. Escobedo, J. Ghiglieri, J. Soto, and A. Vairo, Heavy Quarkonium in a weakly-coupled quark-gluon plasma below the melting temperature, JHEP 1009 (2010) 038, [arXiv: 1007 .4156].

[44] R. Sharma, I. Vitev, and B.-W. Zhang, Light-cone wave function approach to open heavy flavor dynamics in QCD matter, Phys.Rev. C80 (2009) 054902, [arXiv: 0904.0032].

[45] M. Strickland, Thermal Upsilon(1s) and chi 1 suppression in sqrt $(s N N)=2.76 \mathrm{TeV} \mathrm{Pb-Pb}$ collisions at the LHC, Phys.Rev.Lett. 107 (2011) 132301, [arXiv:1106.2571].

[46] A. Emerick, X. Zhao, and R. Rapp, Bottomonia in the Quark-Gluon Plasma and their Production at RHIC and LHC, Eur.Phys.J. A48 (2012) 72, [arXiv:1111.6537].

[47] T. Song, K. C. Han, and C. M. Ko, Bottomonia suppression in heavy-ion collisions, Phys.Rev. C85 (2012) 014902, [arXiv:1109.6691].

[48] G. T. Bodwin, E. Braaten, and G. P. Lepage, Rigorous QCD analysis of inclusive annihilation and production of heavy quarkonium, Phys.Rev. D51 (1995) 1125-1171, [hep-ph/9407339].

[49] N. Borghini and C. Gombeaud, Heavy quarkonia in a medium as a quantum dissipative system: Master equation approach, Eur.Phys.J. C72 (2012) 2000, [arXiv:1109.4271].

[50] N. Dutta and N. Borghini, Sequential suppression of quarkonia and high-energy nucleus-nucleus collisions, arXiv:1206.2149. 